\title{
Hyperbolic angular statistics for globally coupled phase oscillators
}

\author{
M.-O. Hongler (STI/IMT/LPM EPFL - Lausanne - Switzerland) \\ R. Filliger (Bern Applied Univ. - CH-2500 Biel - Switzerland) \\ Ph. Blanchard (Fak. für Physik - Uni Bielefeld - Germany)
}

July 8, 2021

\begin{abstract}
We analytically discuss a multiplicative noise generalization of the Kuramoto-Sakaguchi dynamics for an assembly of globally coupled phase oscillators. In the mean field limit, the resulting class of invariant measures coincides with a generalized, two parameter family of angular von Mises probability distributions which is governed by the exit law from the unit disc of a hyperbolic drifted Brownian motion. Our dynamics offers a simple yet analytically tractable generalization of Kuramoto-Sakaguchi dynamics with two control parameters. We derive an exact and very compact relation between the two control parameters at the onset of phase oscillators synchronization.
\end{abstract}

Keywords: Coupled phase oscillators - Kuramoto dynamics - Generalized von Mises angular distributions - Hyperbolic diffusion processes

PACS number(s): 05.45 Xt - 05.10.Gg - 02.50 Ey

\section{$1 \quad$ Introduction and model}

The modeling efforts aiming to analytically explain the phenomenon of collective synchronization of phase oscillators are, to a large extent, 
based on the Kuramoto model [1, 2] and its mathematical tractable extensions [3]. The success of this "toy model" is thoroughly documented in the recent review by Acebron et al. [4].

Despite a very large body of available literature on coupled phase oscillators, we feel that intimate connections with directional statistics, typically describing distributions on the circle, yet remained relatively neglected. The main purpose of this note is precisely to highlight this intimate connection and explore the fruitful consequences that can be derived from it. As a matter of fact, the mean field stationary solution to the noisy Kuramoto model proposed by Sakaguchi, (referred from now on as the KS model) does on the circle and despite to an apparent non-linearity in the drift term, effectively play the role the Ornstein-Uhlenbeck process plays on the line (see discussion in Mardia [5]). This remark enables to better comprehend why "this little wonder", as the KS model is called in [4] and [6], is analytically tractable. This also suggests that a "non-flat" extension of the mentioned circular Ornstein-Uhenbeck process could be constructed and used to guide us to what we shall call here an hyperbolic extension of the KS model.

Our starting point is a generalized version of the Kuramoto model introduced by Sakaguchi [3]. The model consists of $N$ non-linearly coupled phase oscillators having identical natural frequencies $\omega$ and which are perturbed by multiplicative Gaussian White Noise. The phase of the $i$-th oscillator, denoted by $\theta_{i}$, evolves in time according to

$\dot{\theta}_{i}(t)=\omega+\frac{K}{N} \sum_{j=1}^{N} \sin \left(\theta_{j}-\theta_{i}\right)+\frac{1}{N} \sum_{j=1}^{N}\left\{\sqrt{\left[1+C \cos \left(\theta_{j}-\theta_{i}\right)\right]}\right\} \xi_{i}(t)$.

The first summation over the oscillation population in eq.(11) is a deterministic, non-linear coupling with strength $K>0$. The second summation in eq.(1) couples the oscillators to random fluctuations through the parameter $0<C \leq 1$. The resulting state-dependent noise strength modulates the amplitude of a Gaussian White Noise (WGN) source $\xi_{i}$ 's with constant noise strength $\sqrt{2 T}$ i.e.,

$$
\left\langle\xi_{i}(t)\right\rangle=0 \text { and }\left\langle\xi_{i}(t) \xi_{j}\left(t^{\prime}\right)\right\rangle=2 T \delta_{i j} \delta\left(t-t^{\prime}\right) .
$$

The additional coupling parameter $C$ controls the multiplicative character of the noise source. Indeed, for $C=0$ the additive WGN case is reproduced and eq.(1) therefore coincides with the original KuramotoSakaguchi model.

For $C>0$, we are in presence of multiplicative WGN and the underlying stochastic integral will be interpreted here in the Itô sense.

Observe that the multiplicative noise in eq.(1) itself depends on interactions between the oscillators of the assembly. Several multiplicative 
noise sources for KS dynamics have been previously introduced. In [7, the authors discuss a multiplicative noise KS in which the WGN modulation affecting each oscillator is determined by its dynamical state only, (local noise modulation). A global noise modulation exhibiting similarities with our present model is discussed in [8] where the noise source perturbs the coupling control parameter itself. The [8] dynamics differs from our present model by the fact that in the synchronized states, (i.e. when $\theta_{i}=\theta_{k}, \forall i, k$ ) the dynamics become deterministic a specific feature absent in our model.

In general multiplicative noise sources are known to give rise to new emerging behaviors, the core mechanism for noise induced phase transitions [9]. In the sequel, we shall indeed observe that the onset of synchronized behaviour will be strongly modified when $C>0$.

\section{Self-consistent steady solutions}

As it is usual, we now analyze eqs. (11) and (2) in the mean field approximation reached in the thermodynamic limit $N \rightarrow \infty$. By going into a rotating frame, we can get rid of the common eigenfrequency and hence, we take from now on $\omega=0$. The collection of oscillators can then be characterized by a circular probability density $\rho(\theta, t)$ where $\rho(\theta+d \theta, t)-\rho(\theta, t)$ stands for the fraction of oscillators on the unit circle which, at time $t$, are located at the angular position $\theta$. The circular probability density satisfies the non-linear Fokker-Planck equation

$$
\frac{\partial \rho}{\partial t}=-\frac{\partial}{\partial \theta}[R K \sin (\psi-\theta) \rho]+T \frac{\partial^{2}}{\partial \theta^{2}}[(1+R C \cos (\psi-\theta)) \rho],
$$

with periodic boundary conditions $\rho(\theta+2 \pi, t)=\rho(\theta, t)$ and normalization $\int_{0}^{2 \pi} \rho(\theta, t) d \theta=1$.

In eq.(33), the quantity $R=R(t) \in[0,1]$ yields a measure for the phase coherence of the oscillators and $\psi=\psi(t)$ represents the average phase of the assembly. Both $R(t)$ and $\psi(t)$ are used to construct the complex order parameter

$$
R(t) \exp (i \psi(t)):=\int_{-\pi}^{\pi} e^{i \theta} \rho(\theta, t) d \theta .
$$

Following the original procedure used for the KS model in [1], we use $R$ to monitor the cooperative state of the oscillators assembly. When $R=1$ fully synchronized motion is obtained. The state $R=0$ characterizes the fully incoherent behavior. Intermediate states where $0<R<1$ indicates that the assembly is in a partially synchronized state. 
Under steady state conditions, a self-consistency condition fixes compatible values for $R$ and $\psi$. This ultimately enables to extract the critical coupling strength $K_{c}$ leading to the onset of partially synchronized dynamical states.

For the well known additive noise case $C=0$ the stationary solution to eq. (3) is given by the famous von Mises angular distribution

$$
\rho(\theta)=\frac{e^{k \cos (\psi-\theta)}}{2 \pi I_{0}(k)}
$$

where $I_{0}$ is the modified Bessel function of the first kind and where we introduced the dimensionless factor

$$
k:=R K / T .
$$

Solving self-consistently eq.(4) together with (5) we find the relation:

$$
R=I_{1}(k) / I_{0}(k),
$$

with $I_{1}(z)$ the modified Bessel function of the first kind of order 1 . For the onset of synchronization, i.e. for small but finite $R$, we may expand Eq.(7) to first order in $R$. This yields the well known critical coupling $K_{c}=2 T$ from where partially synchronized solutions branch off the fully incoherent solution represented by the uniform angular distribution $\rho(\theta)=1 / 2 \pi$.

Consider now the generalized, multiplicative noise KS dynamics obtained when $C>0$ in eq.(11). The stationary solution of eq. (3) is given by the so called hyperbolic von Mises distribution (see [10], 11] and the discussion in the next section):

$$
\rho(\theta)=Z^{-1}[1+\tanh (\eta) \cos (\psi-\theta)]^{-\alpha}
$$

with

$$
\tanh (\eta):=R C \text { and } \alpha:=1-\frac{K}{T C} .
$$

The normalization $Z$ in eq. (88) involves the Legendre function $P_{-\alpha}^{(0)}$ of order 0 and degree $-\alpha$ :

$$
Z=2 \pi P_{-\alpha}^{(0)}(\cosh (\eta)) \cosh (\eta)^{\alpha} .
$$

In full analogy with the case $C=0$, we solve self-consistently eq.(41) together with (8) and find the relation (see appendix):

$$
R=\frac{1}{1-\alpha} \frac{P_{-\alpha}^{(1)}(\cosh (\eta))}{P_{-\alpha}^{(0)}(\cosh (\eta))},
$$


where $P_{-\alpha}^{(1)}$ is the Legendre function of order 1 and degree $-\alpha$. To localize the onset of synchronization, we again expand Eq.(99) to first order in $R$, (see appendix) and obtain now the $C$-dependent relation

$$
K_{c}=T(2+C) .
$$

The remarkably simple form of eq.(10), which is our central result, relates the coupling $K$ and the multiplicative noise $C$ constants with the WGN variance $T$. The presence of the extra control parameter $C$ in eq.(10) modifies the phase transition diagram for the synchronization regimes and offers therefore a natural and very simple generalization of the original Kuramoto-Sakaguchi dynamics.

\section{The hyperbolic geometry interpreta- tion of the generalized Kuramoto model}

As mentioned before, the KS model stationary probability measure coincides with the von Mises distribution and is a cornerstone distribution in the theory of directional statistics comparable in many ways with the normal distribution on the line (see Mardia [12]). Among the numerous possibilities to obtain the von Mises distribution eq.(51), let us emphasize here that it describes the exit law from the disc $x^{2}+y^{2} \leq 1$ of a planar Brownian motion starting at the origin subject to a constant drift vector $\mathbf{u}$ of length $k$.

Recently, a very elegant contribution of J.-C. Gruet shows how a twoparameter hyperbolic von Mises distribution $\Lambda^{\alpha}(r, d u)$ can be constructed from the exit law of the hyperbolic Brownian motion with drift $\alpha \in \mathbb{R}$ from the hyperbolic disc of radius $r$, centered at $i,[10$. This hyperbolic extension of the von Mises distribution can be written as

$$
\Lambda^{\alpha}(r, \theta)=\frac{(1+\tanh (r) \cos (\theta))^{-\alpha}}{2 \pi P_{-\alpha}^{(0)}(\cosh (r))}, \quad \theta \in[-\pi, \pi],
$$

which is obviously equivalent to the centered (i.e., $\psi=0$ ) equation (8). This will establish a connection between the generalized KS model eq.(1) and the hyperbolic von Mises distribution.

Observe that independently of Gruets' work the distribution eq.(11) has been recently introduced by Jones and Pewsey as a new family of symmetric distributions on the circle including prominent special cases such as the von Mises distribution, the wrapped Cauchy distribution and others [11]. This family, call them $h_{c}^{\alpha}(r, \theta)$, may be described 
via the $\Lambda^{\alpha}$ 's by coupling $r$ with $\alpha$ through the curvature $-c$ of the hyperbolic space according to the relations $r \rightarrow r c$ and $\alpha \rightarrow \alpha / c$ :

$$
h_{c}^{\alpha}(r, \theta):=\Lambda^{\alpha / c}(r c, \theta), \quad \alpha \in \mathbb{R} \text { and } c \in \mathbb{R}^{+} .
$$

Here $r \geq 0$ stands for a concentration parameter. The index $-c$ can be interpreted as the curvature of the hyperbolic space. As the curvature increases to zero, the usual von Mises distribution is recovered, (see e.g., [1] where they consider the special case $\alpha=1$ ):

$$
\lim _{-c \nearrow_{0}} h_{c}^{\alpha}(r, \theta)=\frac{e^{\alpha r \cos (\theta)}}{2 \pi I_{0}(\alpha r)} .
$$

Now it becomes clear, how to interpret the constant $C>0$ in eq.(11). We have $\eta=R C+\mathcal{O}\left((R C)^{3}\right)$ and $\alpha=1-K / T C$. Hence, small values of $C>0$ produce the following representation for the stationary distribution eq.(8):

$$
\rho(\theta)=h_{C}^{K / T}(R, \theta) .
$$

We then conclude that $-C$ may indeed be interpreted as the curvature of the non-flat manifold underlying the generalized KS model given in eq.(11). Note that the hyperbolic model converges for $C \rightarrow 0$ together with the stationary distribution (8) and the critical coupling (10) to its flat analogue originally discussed in [3].

\section{Conclusions}

It is immediate to realize that the stationary measure of the KuramotoSakaguchi (KS) dynamics coincides with the famous von Mises directional statistics. This distribution on the circle can itself be interpreted as the exit law from a disc of a drifted Brownian motion. An hyperbolic extension, i.e. the exit law of an hyperbolic Brownian motion from a Poincaré disk produces a generalized, two-parameter von Mises probability distribution which has very recently been considered both from a stochastic and a purely statistical point of views. The additional control parameter is intimately connected to the curvature of the underlying non-flat manifold. By analyzing the implication of these features for the KS dynamics, we have been able to construct a simple and natural extension of the original KS model for which, to paraphrase [4] and [6], the "little wonder" remains possible. Our generalization includes, besides the coupling strength between the phase oscillators, a second control parameter, (i.e. the underlying curvature of the curved manifold), which modulates a multiplicative noise source. Ultimately and in full analogy with the original KS model, it is straightforward to derive an exact generalized synchronization diagram which now depends on two control parameters. 


\section{Acknowledgements}

The authors would like to thank partial support from the Centro de Ciencas Mathenaicas (CCM) - Madeira under the project FCT, POCTI-219.

\section{Appendix}

An efficient way to derive eq.(7) is to use the following series representation for the hyperbolic von Mises distribution valid for $\alpha<1$ [10]:

$$
\rho(\theta)=\frac{1}{2 \pi}+\frac{1}{\pi} \sum_{m=1}^{\infty} \frac{\Gamma(1-\alpha)}{\Gamma(m+1-\alpha)} \frac{P_{-\alpha}^{(m)}(\cosh (r))}{P_{-\alpha}^{(0)}(\cosh (r))} \cos (m(\psi-\theta)) .
$$

Replacing the above series in eq.(4) and using a trigonometric identity together with an orthogonality argument for the cosine functions, we find trough elementary integration of $\cos (\theta)$ and $\cos (\theta)^{2}$ over $[-\pi, \pi]$ the equation

$$
R e^{i \psi}=\frac{1}{1-\alpha} \frac{P_{-\alpha}^{(1)}(\cosh (\eta))}{P_{-\alpha}^{(0)}(\cosh (\eta))} e^{i \psi}
$$

which is eq.(7).

Eq.(10) follows from the above by carefully expanding the Legendre functions $P_{-\alpha}^{(0)}$ and $P_{-\alpha}^{(1)}$ to first order in $R$. Recall that $\tanh (\eta)=$ $R C$ and hence $\eta=\frac{1}{2} \ln \left(\frac{1+R C}{1-R C}\right)$. Using in the following the tables in Abramowitz and Stegun [13] we have with 8.11.1:

$$
P_{-\alpha}^{(0)}(\cosh (\eta))=e^{(\alpha-1) \eta} F\left(\frac{1}{2}, 1-\alpha ; 1 ; 1-e^{-2 \eta}\right)
$$

For $P_{-\alpha}^{(1)}$ more care is needed because of a singularity in the Gauss series for the hypergeometric functions $F$. Using 8.11.1 we have

$P_{-\alpha}^{(1)}(\cosh (\eta))=\frac{4 e^{(\alpha-1) \eta}}{1-e^{-2 \eta}} \lim _{\mu \rightarrow 1} \frac{1}{\Gamma(1-\mu)} F\left(-\frac{1}{2},-\alpha ; 1-2 \mu ; 1-e^{-2 \eta}\right)$

The limit is taken using the fact that (see reflection and duplication formula for $\Gamma$-function 6.1 .17 and 6.1 .18 ):

$$
\lim _{\mu \rightarrow 1} \frac{\Gamma(1-2 \mu)}{\Gamma(1-\mu)}=\lim _{\mu \rightarrow 1} \frac{2^{2(1-\mu)-1 / 2}}{\sqrt{2 \pi}(1-2 \mu)} \Gamma\left(1-\mu+\frac{1}{2}\right)=-\frac{1}{2} .
$$


We then have with 15.1.2 in [13:

$P_{-\alpha}^{(1)}(\cosh (\eta))=\frac{4 e^{(\alpha-1) \eta}}{1-e^{-2 \eta}} \frac{\alpha(\alpha-1)}{16}\left(1-e^{-2 \eta}\right)^{2} F\left(\frac{3}{2}, 2-\alpha ; 3 ; 1-e^{-2 \eta}\right)$.

Putting eqs.(17) and (20) into eq.(17) and noting that $1-e^{-2 \eta}=\frac{2 R C}{1+R C}$ we find in first order in $R$

$$
R=-\frac{\alpha}{4} \frac{2 R C}{1+R C}=-\frac{\left(1-\frac{K}{T C}\right)}{4} \frac{2 R C}{1+R C}
$$

which reduces for small but finite $R$ to $K=T(2+C)$.

\section{References}

[1] Kuramoto Y Chemical oscillations, Waves, and Turbulances. Springer, Berlin, 1984, pp. 68-77.

[2] Kuramoto Y. and Nishikawa I. Statistical Macro-dynamics of Large Dynamical Systems. Case of Phase Transitions in Oscillator Communities. J. of Stat. Phys. 49(3,4), (1987), 569-605.

[3] Sakaguchi H. Cooperative phenomena in coupled oscillator system under external fields. Progr. Theor. Phys. 79, (1988), 39.

[4] J.A. Acebron, L. L. Bonilla, C. J. Pérez Vicente, F. Ritort AND R. SPIGler The Kuramoto model: A simple paradigm for synchronization phenomena. Reviews of mod. Phsics, 77 (2005), 137-185.

[5] K.V. Mardia. Statistics of directional data (with discussion). J. Roy. Statist. Soc. Ser. B, 37(472), (1975), 349-393.

[6] S. Strogatz Sability of Incoherence in a Population of Coupled Oscillaters. Journal of Statistical physics, 63 (1991), 613-635.

[7] P. Reimann, C. Van den Broeck, and R. Kawai. Nonequilibrium noise in coupled phase oscillators. PRE 60(6), (1999), 6402-6406.

[8] S.H. Park AND S. KIm Sability of Incoherence in a Population of Coupled Oscillators. Phys. Rev. E, 53 (1996), 3425.

[9] W. Horsthemke and R. Lefever. Noise Induced Phase Transitions. Theory and Applications in Physics, Chemistry and Biology. Springer, second edition, (2006).

[10] Gruet J-C. A note on hyperbolic von Mises distributions. Bernoulli 6(6), (2000), 1007-1020.

[11] M.C. Jones and A. Pewsey. A family of Symmetric Distributions on the Circle. J. of the Amer. Stat. Assoc. 100(472), (2005), 1422-1428.

[12] K.V. Mardia. Statistics of directional data. ACADEMIC PRESS, London, New York, (1972).

[13] M. Abramowitz And I. A. Stegun. Handbook of mathematical functions. National Bureau of Standards Applied Mathematics Series - 55. Tenth Printing, December 1972. 